\title{
Quenched invariance principle for random walks in balanced random environment
}

\author{
Xiaoqin Guo* $\quad$ Ofer Zeitouni ${ }^{\dagger}$
}

April 27, 2010. Revised August 23, 2011

\begin{abstract}
We consider random walks in a balanced random environment in $\mathbb{Z}^{d}, d \geq 2$. We first prove an invariance principle (for $d \geq 2$ ) and the transience of the random walks when $d \geq 3$ (recurrence when $d=2$ ) in an ergodic environment which is not uniformly elliptic but satisfies certain moment condition. Then, using percolation arguments, we show that under mere ellipticity, the above results hold for random walks in i.i.d. balanced environments.
\end{abstract}

\section{Introduction}

In recent years, there has been much interest in the study of invariance principles and transience/recurrence for random walks in random environments (on the $d$-dimensional lattice $\mathbb{Z}^{d}$ ) with non uniformly elliptic transitions probabilities. Much of this work has been in the context of reversible models, either for walks on percolation clusters or for the random conductance model, see [1, 19, 15, 4, 16, 14, 2]. In those cases, the main issue is the transfer of annealed estimates (given e.g. in [6]) to the quenched setting, and the control of the quenched mean displacement of the walk. On the other hand, in these models the reversibility of the walk provides for explicit expressions for certain invariant measures for the environment viewed from the point of view of the particle.

The non-reversible setup has proved to provide many additional, and at this point insurmountable, challenges, even in the uniformly elliptic setup, see [21] for a recent account, and it is therefore premature to study in that

\footnotetext{
* School of Mathematics, University of Minnesota, 206 Church St SE, Minneapolis, MN 55455. Partially supported by NSF grant DMS-0804133.

${ }^{\dagger}$ School of Mathematics, University of Minnesota, 206 Church St SE, Minneapolis, MN 55455 and Faculty of Mathematics, Weizmann Institute, Rehovot 76100, Israel. Partially supported by NSF grant DMS-0804133, the Israel Science Foundation and the Herman P. Taubman chair of Mathematics at the Weizmann Institute
} 
generality the effects of non uniformly elliptic transition probabilities. However, a particular class for which the (quenched) invariance principle has been established in the uniformly elliptic setup is that of walks in balanced environments, see [13]. In that case, a-priori estimates of the AlexandrovBakelman-Pucci type give enough control that allows one to prove the existence of invariant measures (for the environment viewed from the point of view of the particle), and the fact that the walk is a (quenched) martingale together with ergodic arguments yield the invariance principle (obviously, control of the quenched mean displacement, which vanishes, is automatic). The establishment of recurrence (for $d=2$ ) and transience (for $d \geq 3$ ) requires some additional arguments, due to Kesten and Lawler, respectively, see [20] for details.

It is our goal in this paper to explore the extent to which the assumption of uniform ellipticity can be dropped in this non-reversible, but balanced, setup. Not surprisingly, it turns out that some moment assumptions on the ellipticity constant suffice to yield the invariance principle in the ergodic environment setup, after some analytical effort has been expanded in obtaining a-priori estimates. What is maybe more surprising is that for i.i.d. environments, no assumptions of uniform ellipticity are needed at all.

We describe now precisely the model we consider. Let $\mathcal{M}$ be the space of all probability measures on $V=\left\{v \in \mathbb{Z}^{d}:|v| \leq 1\right\}$, where $|\cdot|$ denotes the $l^{2}$ norm. We equip $\mathcal{M}$ with the weak topology on probability measures, which makes it into a Polish space, and equip $\Omega=\mathcal{M}^{\mathbb{Z}^{d}}$ with the induced Polish structure. Let $\mathcal{F}$ be the Borel $\sigma$-field of $\Omega$ and $P$ a probability measure on $\mathcal{F}$.

A random environment is an element $\omega=\{\omega(x, v)\}_{x \in \mathbb{Z}^{d}, v \in V}$ of $\Omega$ with distribution $P$. The random environment is called i.i.d. if $\{\omega(x, \cdot)\}_{x \in \mathbb{Z}^{d}}$ are i.i.d. across the sites $x$ under $P$. The random environment is called balanced if

$$
P\left\{\omega\left(x, e_{i}\right)=\omega\left(x,-e_{i}\right) \text { for all } i \text { and all } x \in \mathbb{Z}^{d}\right\}=1,
$$

and elliptic if $P\left\{\omega(x, e)>0\right.$ for all $|e|=1$ and all $\left.x \in \mathbb{Z}^{d}\right\}=1$.

The random walk in the random environment $\omega \in \Omega$ (RWRE) started at $x$ is the canonical Markov chain $\left\{X_{n}\right\}$ on $\left(\mathbb{Z}^{d}\right)^{\mathbb{N}}$, with state space $\mathbb{Z}^{d}$ and law $P_{\omega}^{x}$ specified by

$$
\begin{aligned}
& P_{\omega}^{x}\left\{X_{0}=x\right\}=1, \\
& P_{\omega}^{x}\left\{X_{n+1}=y+v \mid X_{n}=y\right\}=\omega(y, v), \quad v \in V .
\end{aligned}
$$

The probability distribution $P_{\omega}^{x}$ on $\left(\left(\mathbb{Z}^{d}\right)^{\mathbb{N}}, \mathcal{G}\right)$ is called the quenched law, where $\mathcal{G}$ is the $\sigma$-field generated by cylinder functions. Note that for each $G \in \mathcal{G}, P_{\omega}^{x}(G): \Omega \rightarrow[0,1]$ is a $\mathcal{F}$-measurable function. The joint probability distribution $\mathbb{P}^{x}$ on $\mathcal{F} \times \mathcal{G}$ :

$$
\mathbb{P}^{x}(F \times G)=\int_{F} P_{\omega}^{x}(G) P(\mathrm{~d} \omega), \quad F \in \mathcal{F}, G \in \mathcal{G},
$$


is called the annealed (or averaged) law. Expectations with respect to $P_{\omega}^{x}$ and $\mathbb{P}^{x}$ are denoted by $E_{\omega}^{x}$ and $\mathbb{E}^{x}$, respectively.

Define the canonical shifts $\left\{\theta^{y}\right\}_{y \in \mathbb{Z}^{d}}$ on $(\Omega, \mathcal{F})$ by $\left(\theta^{y} \omega\right)(x, v)=\omega(x+$ $y, v)$. Throughout the paper, we always assume that the system $(\Omega, \mathcal{F}, P)$ is ergodic with respect to the group of shifts $\left\{\theta^{y}\right\}$ and that the environment is balanced and elliptic.

Let $o=(0, \cdots, 0)$ denote the origin and

$$
X_{t}^{n}:=\frac{1}{\sqrt{n}} X_{\lfloor t n\rfloor}+\frac{t n-\lfloor t n\rfloor}{\sqrt{n}}\left(X_{\lfloor t n\rfloor+1}-X_{\lfloor t n\rfloor}\right), \quad t \geq 0 .
$$

We say that the quenched invariance principle holds with nondegenerate covariances if for $P$-almost every $\omega \in \Omega$, the $P_{\omega}^{o}$ law of the path $\left\{X_{t}^{n}\right\}_{t \geq 0}$ converges weakly to a Brownian motion on $\mathbb{R}^{d}$ with covariance matrix $\left(a_{i} \delta_{i j}\right)_{1 \leq i, j \leq d}$, $a_{i}>0$, as $n \rightarrow \infty$.

Lawler [13] proved a quenched invariance principle for random walks in a balanced random environment under the assumption that the random environment is uniformly elliptic, i.e.

$$
P\left\{\omega(x, e) \geq \varepsilon_{0} \text { for all }|e|=1\right\}=1 \quad \text { for some } \varepsilon_{0}>0 .
$$

As mentioned above, our goal in this paper is to study the extent to which the uniform ellipticity assumption can be dropped. Let

$$
\varepsilon(x)=\varepsilon_{\omega}(x):=\left[\prod_{i=1}^{d} \omega\left(x, e_{i}\right)\right]^{\frac{1}{d}} .
$$

Our first main result is that if $\operatorname{E} \varepsilon(o)^{-p}<\infty$ for some $p>d$, then the quenched invariance principle holds and moreover, the RWRE is transient $P$-almost surely if $d \geq 3$. (Recurrence for $d=2$ under the condition $E \varepsilon(0)^{-p}<\infty$ follows from the quenched invariance principle and ergodicity by an unpublished argument of Kesten detailed in [20, Page 281]. Note that this argument cannot be used to prove transience in dimensions $d \geq 3$, even given an invariance principle, since in higher dimensions the invariance principle does not give useful information on the range of the random walk; the behavior of the range is a crucial element in Kesten's argument.)

Theorem 1. Assume that the random environment is ergodic, elliptic and balanced.

(i) If $E \varepsilon(o)^{-p}<\infty$ for some $p>d \geq 2$, then the quenched invariance principle holds with a nondegenerate limiting covariance.

(ii) If $E[(1-\omega(o, o)) / \varepsilon(o)]^{q}<\infty$ for some $q>2$ and $d \geq 3$, then the $R W R E$ is transient P-almost surely. 
That some integrability condition on the tail of $\varepsilon(o)$ is needed for part (i) to hold is made clear by the (non-Gaussian) scaling limits of random walks in Bouchaud's trap model, see [5, 3]. In fact, it follows from that example that Theorem 1(i), or even an annealed version of the CLT, cannot hold in general with $p<1$.

The proof of Theorem 1 is based on a sharpening of the arguments in [13, 18, 20]; in particular, refined versions of the maximum principle for walks in balanced environments (Theorem 3) and of a mean value inequality (Theorem 12) play a crucial role.

When the environment is i.i.d. and elliptic, our second main result is that if $\left|X_{n+1}-X_{n}\right|=1$ a.s., then the quenched invariance principle holds. Moreover, the RWRE is $P$-almost surely transient when $d \geq 3$. The proofs combine percolation arguments with Theorem 1.

Theorem 2. Assume that the random environment is i.i.d., elliptic and balanced.

(i) If $P\left\{\max _{|e|=1} \omega(o, e) \geq \xi_{0}\right\}=1$ for some positive constant $\xi_{0}$, then the quenched invariance principle holds with a non-degenerate limiting covariance.

(ii) When $d \geq 3$, the RWRE is transient P-almost surely.

Because the transience or recurrence of the random walks does not change if one considers the walk restricted to its jump times, one concludes, using Kesten's argument and the invariance principle, compare with Theorem 1, that for $d=2$, a random walk in a balanced elliptic i.i.d. random environment is recurrent $P$-a.s.

Our proof of the invariance principles, like that of [13], is based on the approach of the "environment viewed from the point of view of the particle". Specifically, set $\bar{\omega}(n)=\theta^{\mathrm{X}_{n}} \omega$, then the process $\bar{\omega}(n)$ is a Markov chain under $\mathbb{P}^{o}$ with state space $\Omega$ and transition kernel

$$
M\left(\omega^{\prime}, \mathrm{d} \omega\right)=\sum_{i=1}^{d}\left[\omega^{\prime}\left(o, e_{i}\right) \delta_{\theta^{e} i \omega^{\prime}}+\omega^{\prime}\left(o,-e_{i}\right) \delta_{\theta^{-e_{i} \omega^{\prime}}}\right]+\omega^{\prime}(o, o) \delta_{\omega^{\prime}} .
$$

Since $\left\{X_{n}\right\}$ is a (quenched) martingale, standard arguments (see the proof of Theorem 6.2 in [4]) show that the quenched invariance principle holds whenever an invariant measure $Q \sim P$ of $\{\bar{\omega}(n)\}$ exists. The approach of Lawler [13], which is a discrete version of the argument of Papanicolaou and Varadhan [17, is to construct such a measure as the limit of invariant measures of periodized environments. We will follow this strategy using, as in [18, 20], variants of [11] to derive estimates on solutions of linear elliptic difference equations. In the i.i.d. setup of Theorem 2, percolation estimates are used to control pockets of the environment where those estimates are 
not strong enough.

For the proof of the transience in the ergodic case, we use a mean value inequality and follow [20]. To prove the transience in the iid case, we employ percolation arguments together with a new maximum principle (Theorem 15) for walks with (possibly) big jumps.

The structure of this paper is as follows. In Section 2 we construct the "periodized environments" as in [18, 20], and show that the proof of $Q \sim P$ can be reduced to the proof of the inequality (3). Using the maximum principle, we then prove (3) in Section 3 under the assumptions of Theorem 1(i). In Section 4, devoted to the i.i.d. setup, we prove Theorem 2(i), using percolation tools. Section 5 is devoted to the proof of the transience of the RWRE for $d \geq 3$, thus providing a proof of Theorem 1(ii). In Section 6, we will show a modified maximum principle for balanced difference operators, and use it to prove Theorem 2(ii).

Throughout the paper, $C$ denotes a generic positive constant, that may depend on dimension only, and whose value may change from line to line.

\section{The periodized environments}

As in [18, 20], the following periodic structure of the environment is introduced.

Let $\Delta_{N}\left(x_{0}\right)=\left\{x \in \mathbb{Z}^{d}:\left|x-x_{0}\right|_{\infty} \leq N\right\}$ be the cube centered at $x_{0}$ of length $2 N$. Let $\Delta_{N}=\Delta_{N}(o)$. For any $x \in \mathbb{Z}^{d}$, set

$$
\hat{x}:=x+(2 N+1) \mathbb{Z}^{d} \in \mathbb{Z}^{d} /(2 N+1) \mathbb{Z}^{d} .
$$

For any fixed $\omega \in \Omega$, we define $\omega^{N}$ by setting $\omega^{N}(x)=\omega(x)$ for $x \in \Delta_{N}$ and $\omega^{N}(y)=\omega^{N}(x)$ for $y \in \mathbb{Z}^{d}$ whenever $\hat{y}=\hat{x}$. Let $\Omega^{N}=\left\{\omega^{N}: \omega \in\right.$ $\Omega\}$. Let $\left\{X_{n, N}\right\}$ denote the random walk on $\mathbb{Z}^{d}$ in the environment $\omega^{N}$. Then $\left\{\hat{X}_{n, N}\right\}$ is an irreducible finite-state Markov chain, hence it possesses a unique invariant probability measure, which can always be written in the form

$$
\frac{1}{(2 N+1)^{d}} \sum_{x \in \Delta_{N}} \Phi_{N}(x) \delta_{\hat{x}}
$$

Here $\Phi_{N}$ is some function on $\Delta_{N}$ and $(2 N+1)^{-d} \Phi_{N}(\cdot)$ sums to 1 , so that $\Phi_{N}$ can be interpreted as a density with respect to the uniform measure on $\Delta_{N}$.

Define

$$
Q_{N}=Q_{N, \omega}=\frac{1}{(2 N+1)^{d}} \sum_{x \in \Delta_{N}} \Phi_{N}(x) \delta_{\theta^{x} \omega^{N}}
$$


as a probability measure on $\Omega^{N}$. Then for any $x \in \Delta_{N}$,

$$
\begin{aligned}
\sum_{y \in \Delta_{N}} Q_{N}\left(\theta^{y} \omega^{N}\right) M\left(\theta^{y} \omega^{N}, \theta^{x} \omega^{N}\right) & =\sum_{y \in \Delta_{N}} \frac{\Phi_{N}(y)}{(2 N+1)^{d}} \omega^{N}(y, x) \\
& =\frac{\Phi_{N}(x)}{(2 N+1)^{d}}=Q_{N}\left(\theta^{x} \omega^{N}\right) .
\end{aligned}
$$

This implies that $Q_{N}$ is the invariance probability measure (with respect to the kernel $M$ ) for the Markov chain $\left\{\bar{\omega}^{N}(n)\right\}$ on $\Omega^{N}$.

We will show that $Q_{N}$ converges weakly to some measure $Q$ with good properties. To do this, we first introduce a sequence of measures

$$
P_{N}=P_{N, \omega}=\frac{1}{(2 N+1)^{d}} \sum_{x \in \Delta_{N}} \delta_{\theta^{x} \omega^{N}}
$$

which by the multidimensional ergodic theorem (see Theorem (14.A8) in [8] and also Theorem 1.7.5 in [10]) converges weakly to $P, P$ - a.s.

Let $\left\{\omega_{\gamma}^{N}\right\}_{\gamma=1}^{k}$ denote the set of distinct states in $\left\{\theta^{x} \omega^{N}\right\}_{x \in \Delta_{N}}$ and $C_{N}(\gamma):=$ $\left\{x \in \Delta_{N}: \theta^{x} \omega^{N}=\omega_{\gamma}^{N}\right\}$. Set, for any finite subset $E \subset \mathbb{Z}^{d}$,

$$
\|f\|_{E, j}:=\left(|E|^{-1} \sum_{x \in E}|f(x)|^{j}\right)^{\frac{1}{j}} .
$$

Since $\mathrm{d} Q_{N} / \mathrm{d} P_{N}=\sum_{\gamma=1}^{k} \delta_{\omega_{\gamma}^{N}}\left|C_{N}(\gamma)\right|^{-1} \sum_{x \in C_{N}(\gamma)} \Phi_{N}(x):=f_{N}$, we have that for any measurable function $g$ on $\Omega$,

$$
\begin{aligned}
\left|Q_{N} g\right| & \leq\left(\int f_{N}^{\alpha} \mathrm{d} P_{N}\right)^{\frac{1}{\alpha}}\left(\int|g|^{\alpha^{\prime}} \mathrm{d} P_{N}\right)^{\frac{1}{\alpha^{\prime}}} \\
& \leq\left(\frac{1}{\left|\Delta_{N}\right|} \sum_{\gamma=1}^{k} \sum_{x \in C_{N}(\gamma)} \Phi_{N}(x)^{\alpha}\right)^{\frac{1}{\alpha}}\left(\int|g|^{\alpha^{\prime}} \mathrm{d} P_{N}\right)^{\frac{1}{\alpha^{\prime}}} \\
& =\left\|\Phi_{N}\right\|_{\Delta_{N}, \alpha}\left(P_{N}|g|^{\alpha^{\prime}}\right)^{\frac{1}{\alpha^{\prime}}},
\end{aligned}
$$

where $\alpha^{\prime}$ is the Hölder conjugate of $\alpha, 1 / \alpha+1 / \alpha^{\prime}=1$, and we used Hölder's inequality in the first and the second inequalities. Since $\Omega$ is compact with respect to the product topology, along some subsequence $N_{k} \rightarrow \infty,\left\{Q_{N_{k}}\right\}$ converges weakly to a limit, denoted $Q$. Assume for the moment that

$$
\varlimsup_{N \rightarrow \infty}\left\|\Phi_{N}\right\|_{\Delta_{N}, \alpha} \leq C, \quad P-\text { a.s.. }
$$

We show that then, for a.e. $\omega \in \Omega$,

$$
Q \ll P .
$$

Indeed, let $A \subset \Omega$ be measurable. Let $\rho$ denote a metric on the Polish space $\Omega$. For any closed subset $F \subset A, \delta>0$, introduce the function 
$f(\omega)=[1-\rho(\omega, F) / \delta]^{+}$which is supported on $F_{\delta}=\{\omega \in \Omega: \rho(\omega, F)<\delta\}$. Then by (2), (3),

$$
Q F \leq \varlimsup_{N \rightarrow \infty} Q_{N} f \leq C\left(P f^{\alpha^{\prime}}\right)^{\frac{1}{\alpha^{\prime}}} \leq C\left(P F_{\delta}\right)^{\frac{1}{\alpha^{\prime}}} .
$$

Letting $\delta \downarrow 0$, we get $Q F \leq C(P F)^{\frac{1}{\alpha^{\prime}}}$. Taking supremums over all closed subset $F \subset A$, one concludes that $Q A \leq C \cdot(P A)^{\frac{1}{\alpha^{\prime}}}$, which proves (4).

Once we have (4), it is standard to check, using ellipticity, that $\bar{\omega}(n)$ is ergodic with respect to $Q$ and $Q \sim P$ (see [18, 20]). (Thus, by the ergodic theorem, $Q$ is uniquely determined by $Q g=\lim _{n \rightarrow \infty} E \sum_{j=0}^{n-1} g\left(\bar{\omega}_{j}\right) / n$ for every bounded measurable $g$. Hence $Q$ is the weak limit of $Q_{N}$.) Therefore, to prove the invariance principle it suffices to prove (3). Sections 3 and Section 4 are devoted to the proof of (3), under the assumptions of Theorems 1 and 2 ,

\section{Maximum Principle and proof of Theorem 1)(i)}

Throughout this section, we fix an $\omega \in \Omega$. For any bounded set $E \subset \mathbb{Z}^{d}$, let $\partial E=\left\{y \in E^{c}: \exists x \in E,|x-y|_{\infty}=1\right\}, \bar{E}=E \bigcup \partial E$ and $\operatorname{diam}(E)=$ $\max \left\{|x-y|_{\infty}: x, y \in E\right\}$. For any function $f$ defined on $\bar{E}$, let $L_{\omega}$ denote the operator

$$
\left(L_{\omega} f\right)(x)=\sum_{i=1}^{d} \omega\left(x, e_{i}\right)\left[f\left(x+e_{i}\right)+f\left(x-e_{i}\right)-2 f(x)\right], \quad x \in E .
$$

The following discrete maximum principle is an adaption of Theorem 2.1 of 11 .

Theorem 3 (Maximum Principle). Let $E \subset \mathbb{Z}^{d}$ be bounded, and let $u$ be a function on $\bar{E}$. For all $x \in E$, assume $\varepsilon(x)>0$ and define

$$
I_{u}(x):=\left\{s \in \mathbb{R}^{d}: u(x)-s \cdot x \geq u(z)-s \cdot z, \forall z \in \bar{E}\right\} .
$$

If $L_{\omega} u(x) \geq-g(x)$ for all $x \in E$ such that $I_{u}(x) \neq \emptyset$, then

$$
\max _{E} u \leq C \operatorname{diam} \bar{E}\left(\sum_{\substack{x \in E \\ I_{u}(x) \neq \emptyset}}\left|\frac{g}{\varepsilon}\right|^{d}\right)^{\frac{1}{d}}+\max _{\partial E} u .
$$

In particular,

$$
\max _{E} u \leq C \operatorname{diam} \bar{E} \cdot|E|^{\frac{1}{d}}\left\|\frac{g}{\varepsilon}\right\|_{E, d}+\max _{\partial E} u
$$


Proof: See the proof of Theorem 2.1 in [11].

Define the stopping times $\tau_{0}=0, \tau_{1}=\tau:=\min \left\{j \geq 1: \mid X_{j, N}-\right.$ $\left.\left.X_{0, N}\right|_{\infty}>N\right\}$ and $\tau_{j+1}=\min \left\{n>\tau_{j}:\left|X_{n, N}-X_{\tau_{j}, N}\right|_{\infty}>N\right\}$.

Lemma 4. Let $\omega^{N},\left\{X_{n, N}\right\}$ be as in Section 1 and $\tau$ as defined above, then there exists a constant $c$ such that, for all $N$ large,

$$
E_{\theta^{x} \omega^{N}}^{o}\left(1-\frac{c}{N^{2}}\right)^{\tau} \leq C<1
$$

Proof: Since $\mathrm{P}$ is balanced, $X_{n, N}$ is a martingale and it follows from Doob's inequality that for any $K \geq 1$,

$$
\begin{aligned}
P_{\theta^{x} \omega^{N}}^{o}\{\tau \leq K\} & \leq 2 \sum_{i=1}^{d} P_{\theta^{x} \omega^{N}}^{o}\left\{\sup _{n \leq K} X_{n, N}(i) \geq N+1\right\} \\
& \leq \frac{2}{N+1} \sum_{i=1}^{d} E_{\theta^{x} \omega^{N}}^{o} X_{K, N}(i)^{+} \leq \frac{2 d}{N+1} \sqrt{K}
\end{aligned}
$$

where $X_{n, N}(i)$ is the $i$-th coordinate of $X_{n, N}$. Hence

$$
E_{\theta^{x} \omega^{N}}^{o}\left(1-\frac{c}{N^{2}}\right)^{\tau} \leq\left(1-\frac{c}{N^{2}}\right)^{K}+\frac{2 d}{N+1} \sqrt{K} .
$$

Taking $c=16 d^{2}$ and $K=N^{2} / 16 d^{2}$, we get $E_{\theta^{x} \omega^{N}}^{o}\left(1-\frac{c}{N^{2}}\right)^{\tau} \leq e^{-1}+2^{-1}$.

\section{Theorem 5.}

$$
\left\|\Phi_{N} \varepsilon\right\|_{\Delta_{N}, \beta} \leq C
$$

where $\beta=d^{\prime}=d /(d-1)$.

Proof: Let $c$ be the same constant as in the previous lemma. For any function $h \geq 0$ on $\Delta_{N}$,

$$
\begin{aligned}
& \left\|\Phi_{N} \cdot h\right\|_{\Delta_{N}, 1} \\
& =\frac{c}{N^{2}} \sum_{x \in \Delta_{N}} \frac{\Phi_{N}(x)}{\left|\Delta_{N}\right|} \sum_{m \geq 0} E_{\omega^{N}}^{x} \sum_{\tau_{m} \leq j<\tau_{m+1}}\left(1-\frac{c}{N^{2}}\right)^{j} h\left(\hat{X}_{j, N}\right) \\
& \leq \frac{c}{N^{2}} \sum_{x \in \Delta_{N}} \frac{\Phi_{N}(x)}{\left|\Delta_{N}\right|} \sum_{m \geq 0} E_{\omega^{N}}^{x}\left(1-\frac{c}{N^{2}}\right)^{\tau_{m}} E_{\omega^{N}}^{\hat{X}_{\tau_{m}, N}} \sum_{j=0}^{\tau-1} h\left(\hat{X}_{j, N}\right) \\
& \leq \frac{c}{N^{2}} \sum_{x \in \Delta_{N}} \frac{\Phi_{N}(x)}{\left|\Delta_{N}\right|} \sum_{m \geq 0}\left[\sup _{y \in \Delta_{N}} E_{\omega^{N}}^{y}\left(1-\frac{c}{N^{2}}\right)^{\tau}\right]^{m} \cdot \sup _{y \in \Delta_{N}} E_{\omega^{N}}^{y} \sum_{j=0}^{\tau-1} h\left(\hat{X}_{j, N}\right) .
\end{aligned}
$$

Since the function $f(x)=E_{\omega^{N}}^{x} \sum_{j=0}^{\tau-1} h\left(\hat{X}_{j, N}\right)$ satisfies

$$
\left\{\begin{aligned}
L_{\omega^{N}} f(x)=h(x), & \text { if } x \in \Delta_{N} \\
f(x)=0, & \text { if } x \in \partial \Delta_{N},
\end{aligned}\right.
$$


we can apply the maximum principle (Theorem [3) and get

$$
\sup _{y \in \Delta_{N}} E_{\omega^{N}}^{y} \sum_{j=0}^{\tau-1} h\left(\hat{X}_{j, N}\right) \leq C N^{2}\left\|\frac{h}{\varepsilon}\right\|_{\Delta_{N}, d} .
$$

This together with Lemma 4 and $\sum_{x \in \Delta_{N}} \Phi_{N}(x) /\left|\Delta_{N}\right|=1$ yield

$$
\left\|\Phi_{N} \cdot h\right\|_{\Delta_{N}, 1} \leq C\left\|\frac{h}{\varepsilon}\right\|_{\Delta_{N}, d}
$$

Hence by the duality of norms,

$$
\left\|\Phi_{N} \varepsilon\right\|_{\Delta_{N}, \beta}=\sup _{\left\|\frac{h}{\varepsilon}\right\|_{\Delta_{N}, d}=1}\left\|\Phi_{N} h\right\|_{\Delta_{N}, 1} \leq C .
$$

Proof of (3) under the assumption of Theorem 1(i):

Assume that

$$
\mathrm{E} \varepsilon(o)^{-p}<\infty \text { for some } p>d .
$$

Take $\alpha=(1-1 / d+1 / p)^{-1}$. We use Hölder's inequality and Theorem 5 to get

$$
\left\|\Phi_{N}\right\|_{\Delta_{N}, \alpha} \leq\left\|\Phi_{N} \varepsilon\right\|_{\Delta_{N}, \beta}\left\|\varepsilon^{-1}\right\|_{\Delta_{N}, p} \leq C\left\|\varepsilon^{-1}\right\|_{\Delta_{N}, p} .
$$

By the multidimensional ergodic theorem,

$$
\lim _{N \rightarrow \infty}\left\|\varepsilon^{-1}\right\|_{\Delta_{N}, p}=\left(E \varepsilon(o)^{-p}\right)^{\frac{1}{p}}<\infty, \quad P \text { - a.s.. }
$$

Remark 6. Without the assumption (8), the conclusion (3) may fail. To see the difficulty, let

$$
A=A\left(\omega, \varepsilon_{0}\right)=\left\{x: \min _{i} \omega\left(x, e_{i}\right)<\varepsilon_{0}\right\} .
$$

By (6) we have

$$
\left\|\Phi_{N} 1_{A^{c}}\right\|_{\Delta_{N}, \beta} \leq\left\|\Phi_{N} \frac{\varepsilon}{\varepsilon_{0}}\right\|_{\Delta_{N}, \beta} \leq \frac{C}{\varepsilon_{0}} .
$$

In order to proceed as before, we need to show that $\overline{\lim }_{N \rightarrow \infty}\left\|\Phi_{N} 1_{A}\right\|_{\Delta_{N}, \alpha} \leq C$ for some $1<\alpha \leq \beta$. As Bouchaud's trap model [5, 3] shows, this is not always the case. However, if $P\left\{\max _{|e|=1} \omega(o, e) \geq \xi_{0}\right\}=1$, then for $x \in A$, we have, using that the environment is balanced, some control of $\Phi_{N}(x)$ by $\left.\Phi_{N}\right|_{A^{c}}$ (see Lemma 7). Further, in the i.i.d. case, A corresponds to a 'site percolation' model, whose cluster sizes can be estimated. We will show in the next section that these properties lead to a proof of (3) in the i.i.d. setup, without moment assumptions. 


\section{A percolation estimate and proof of Theorem 2(i)}

In this section we consider the RWRE in the i.i.d. setting where $\max _{|e|=1} \omega(x, e) \geq$ $\xi_{0}$ for all $x \in \mathbb{Z}^{d}$ and all $\omega \in \Omega$. We begin by introducing some terminology.

The $l^{1}$-distance (graph distance) from $x$ to $y$ is defined as

$$
d(x, y)=|x-y|_{1}=\sum_{i=1}^{d}\left|x_{i}-y_{i}\right| .
$$

Note that $|x|_{\infty} \leq|x|_{1} \leq d|x|_{\infty}$.

In an environment $\omega$, we say that a site $x$ is open(closed) if $\min _{i} \omega\left(x, e_{i}\right)<$ $\varepsilon_{0}\left(\geq \varepsilon_{0}\right.$, resp. $)$ and that an edge of $\mathbb{Z}^{d}$ is open if its endpoints are open. Here $\varepsilon_{0}>0$ is a constant whose value is to be determined. An edge is called closed if it is not open. Let $A=A(\omega)$ denote the subgraph of $\mathbb{Z}^{d}$ obtained by deleting all closed edges and closed sites. We call $A(\omega)$ a site percolation with parameter $p=p\left(\varepsilon_{0}\right)=P\left\{\min _{i} \omega\left(x, e_{i}\right)<\varepsilon_{0}\right\}$.

A percolation cluster is a connected component of $A$. (Although here a percolation cluster is defined as a graph, we also use it as a synonym for its set of vertices.) The $l^{1}$ diameter of a percolation cluster $B$ is defined as $l(B)=\sup _{x \in B, y \in \partial B} d(x, y)$. For $x \in A$, let $A_{x}$ denote the percolation cluster that contains $x$ and let $l_{x}$ denote its diameter. Set $A_{x}=\emptyset$ and $l_{x}=0$ if $x \notin A$. We let $\varepsilon_{0}$ be small enough such that $l_{x}<\infty$ for all $x \in \mathbb{Z}^{d}$.

We call a sequence of sites $\left(x^{1}, \cdots, x^{n}\right)$ a path from $x$ to $y$ if $x^{1}=x$, $x^{n}=y$ and $\left|x^{j}-x^{j+1}\right|=1$ for $j=1, \cdots, n-1$. Let

$$
\square=\left\{\left(\kappa_{1}, \cdots, \kappa_{d}\right) \in \mathbb{Z}^{d}: \kappa_{i}= \pm 1\right\} .
$$

We say that a path $\left\{x^{1}, \cdots, x^{n}\right\}$ is a $\kappa$-path, $\kappa \in \square$, if

$$
\omega\left(x^{j}, x^{j+1}-x^{j}\right) \geq \xi_{0}
$$

and $\kappa_{i}\left(x^{j+1}-x^{j}\right)_{i} \geq 0$ for all $i=1, \cdots, d$ and $j=1, \cdots, n-1$. Observing that for each site there exist at least two neighbors (in opposite directions) to whom the transition probabilities are $\geq \xi_{0}$, we have the following property concerning the structure of the balanced environment:

- For any $x \in A$ and any $\kappa \in \square$, there exists a $\kappa$-path from $x$ to some $y \in \partial A_{x}$, and this path is contained in $\bar{A}_{x}$.

This property gives us a useful inequality.

Lemma 7. For $x \in A \cap \Delta_{N}$, if $l_{x} \leq N$, then

$$
\Phi_{N}(x) \leq \xi_{0}^{-l_{x}} \sum_{y \in \partial A_{x} \cap \Delta_{N}} \Phi_{N}(y) .
$$


Proof: Suppose that $A_{x} \neq \emptyset$ (otherwise the proof is trivial). Since $l_{x} \leq N$, $\bar{A}_{x} \subset \Delta_{N}(x)$. Note that at least one of the $2^{d}$ corners of $\Delta_{N}(x)$ is contained in $\Delta_{N}$. Without loss of generality, suppose that $v=x+(N, \cdots, N) \subset \Delta_{N}$. Then there is a $(1, \cdots, 1)$-path in $\bar{A}_{x}$ from $x$ to some $y \in \partial A_{x} \cap \Delta_{N}$, as illustrated in the following figure:

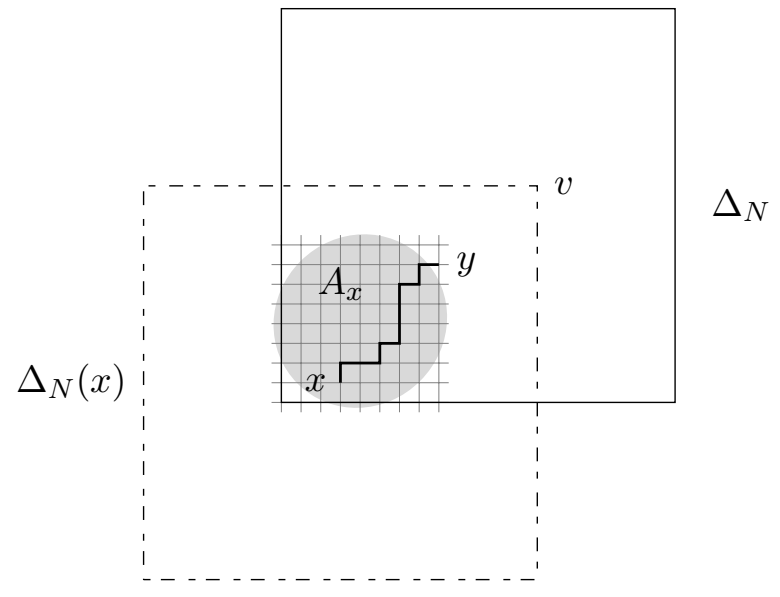

Recalling that $\Phi_{N}$ is the invariant measure for $\left\{\hat{X}_{n, N}\right\}$ defined in Section 1 , we have

$$
\begin{aligned}
\Phi_{N}(y) & =\sum_{z \in \Delta_{N}} \Phi_{N}(z) P_{\omega^{N}}^{d(x, y)}(\hat{z}, \hat{y}) \\
& \geq \Phi_{N}(x) P_{\omega^{N}}^{d(x, y)}(\hat{x}, \hat{y}) \geq \Phi_{N}(x) \xi_{0}^{l_{x}} .
\end{aligned}
$$

Here $P_{\omega^{N}}^{m}(\hat{z}, \hat{y})$ denotes the $m$-step transition probability of $\left\{\hat{X}_{n, N}\right\}$ from $\hat{z}$ to $\hat{y}$.

Let $S_{n}=\left\{x:|x|_{\infty}=n\right\}$ denote the boundary of $\Delta_{n}$. Let $x \rightarrow y$ be the event that $y \in \bar{A}_{x}$ and $o \rightarrow S_{n}$ be the event that $o \rightarrow x$ for some $x \in S_{n}$. The following theorem, which is the site percolation version of the combination of Theorems 6.10 and 6.14 in [9], gives an exponential bound on the diameter of the cluster containing the origin, when $p$ is small.

Theorem 8. There exists a function $\varphi(p)$ of $p=p\left(\varepsilon_{0}\right)$ such that

$$
P\left\{o \rightarrow S_{n}\right\} \leq C n^{d-1} e^{-n \varphi(p)}
$$

and $\lim _{p \rightarrow 0} \varphi(p)=\infty$.

Let $A_{x}(n)$ denote the connected component of $A_{x} \cap \Delta_{n}(x)$ that contains $x$ and set

$$
q_{n}=P\left\{o \rightarrow S_{n}\right\} .
$$

The proof of Theorem 8 will proceed by showing some (approximate) subadditivity properties of $q_{n}$. We thus recall Fekete's subadditivity lemma ([7]): 
Lemma 9. If a sequence of finite numbers $\left\{b_{k}: k \geq 1\right\}$ is subadditive, that $i s, b_{m+n} \leq b_{m}+b_{n}$ for all $m, n$, then $\lim _{k \rightarrow \infty} b_{k} / k=\inf _{k \in \mathbb{N}} b_{k} / k$.

Proof of Theorem 8: We follow the proof given by Grimmett in [9] in the bond percolation case. By the BK inequality (9], pg. 38),

$$
q_{m+n} \leq \sum_{x \in S_{m}} P\{o \rightarrow x\} P\left\{x \rightarrow x+S_{n}\right\} .
$$

But $P\{o \rightarrow x\} \leq q_{m}$ for $x \in S_{m}$ and $P\left\{x \rightarrow x+S_{n}\right\}=q_{n}$ by translation invariance. Hence we get

$$
q_{m+n} \leq\left|S_{m}\right| q_{m} q_{n}
$$

By exchanging $m$ and $n$ in (10),

$$
q_{m+n} \leq\left|S_{m \wedge n}\right| q_{m} q_{n} .
$$

On the other hand, let $U_{x}$ be the event that $x \in \overline{A_{o}(m)}$ and let $V_{x}$ be the event that $\overline{A_{x}(n)} \cap S_{m+n} \neq \emptyset$. We use the FKG inequality ([9], pg. 34) to find that

$$
q_{m+n} \geq P\left\{U_{x}\right\} P\left\{V_{x}\right\} \quad \text { for any } x \in S_{m} .
$$

However, $\sum_{x \in S_{m}} P\left\{U_{x}\right\} \geq q_{m}$, which implies that

$$
\max _{x \in S_{m}} P\left\{U_{x}\right\} \geq \frac{q_{m}}{\left|S_{m}\right|} .
$$

Let $\gamma_{n}=P\left\{\overline{A_{o}(n)} \cap\left\{x: x_{1}=n\right\} \neq \emptyset\right\}$, then $P\left\{V_{x}\right\} \geq \gamma_{n}$. Moreover, $\gamma_{n} \leq q_{n} \leq 2 d \gamma_{n}$. Hence

$$
q_{m+n} \geq \frac{q_{m} q_{n}}{2 d\left|S_{m}\right|},
$$

and then

$$
q_{m+n} \geq \frac{q_{m} q_{n}}{2 d\left|S_{m \wedge n}\right|} .
$$

Note that $\left|S_{m}\right| \leq C_{d} m^{d-1}$. Letting

$$
b_{k}=\log q_{k}+\log C_{d}+(d-1) \log (2 k),
$$

one checks using (11) that the sequence $\left\{b_{k}\right\}$ is subadditive. Similarly by (12), $\left\{-\log q_{k}+\log \left(2 d C_{d}\right)+(d-1) \log (2 k)\right\}$ is subadditive. Thus, using Lemma 9 ,

$$
\varphi(p):=-\lim _{k \rightarrow \infty} \frac{1}{k} \log q_{k}
$$

exists and

$$
\begin{gathered}
\log q_{k}+\log C_{d}+(d-1) \log (2 k) \geq-k \varphi(p) \\
-\log q_{k}+\log \left(2 d C_{d}\right)+(d-1) \log (2 k) \geq k \varphi(p) .
\end{gathered}
$$

The first part of the theorem follows simply from (14), and the second by noting that with $p \downarrow 0$ in (13) we have $q_{k} \downarrow 0$ and then $\varphi(p) \rightarrow \infty$. 
Remark 10. It follows from Theorem 8 that

$$
P\left\{l_{o} \geq n\right\} \leq P\left\{o \rightarrow S_{\lfloor n / 2 d\rfloor}\right\} \leq C e^{\varphi(p)} n^{d-1} e^{-n \varphi(p) / 2 d} .
$$

With (15) and the Borel-Cantelli lemma one concludes that P-almost surely, $l_{x} \leq N$ is true for all $x \in \Delta_{N}$ when $N$ is sufficiently large and $p$ is such that $\varphi(p)>0$. Hence the inequality (9) holds for all $x \in \Delta_{N}$ when $N$ is large.

Proof of (3) under the assumption of Theorem Q(i): By Hölder's inequality,

$$
\frac{1}{\left|\Delta_{N}\right|} \sum_{y \in \partial A_{x} \cap \Delta_{N}} \Phi(y) \leq\left\|\Phi_{N} 1_{\partial A_{x}}\right\|_{\Delta_{N}, \beta}\left(\frac{\left|\partial A_{x}\right|}{\left|\Delta_{N}\right|}\right)^{1-1 / \beta},
$$

so when $N$ is large enough we have by Lemma 7 that for any $x \in A \cap \Delta_{N}$,

$$
\Phi_{N}(x) \leq \xi_{0}^{-l_{x}}\left|\partial A_{x}\right|^{1-1 / \beta}\left|\Delta_{N}\right|^{1 / \beta}\left\|\Phi_{N} 1_{\partial A_{x}}\right\|_{\Delta_{N}, \beta} .
$$

Hence for any $\alpha \in(1, \beta)$,

$$
\begin{aligned}
\left\|\Phi_{N} 1_{A}\right\|_{\Delta_{N}, \alpha}^{\alpha} & \frac{1}{\left|\Delta_{N}\right|} \sum_{x \in A \cap \Delta_{N}}\left(\xi_{0}^{-l_{x}}\left|\partial A_{x}\right|^{1-1 / \beta}\left|\Delta_{N}\right|^{1 / \beta}\left\|\Phi_{N} 1_{\partial A_{x}}\right\|_{\Delta_{N}, \beta}\right)^{\alpha} \\
\leq & {\left[\frac{1}{\left|\Delta_{N}\right|} \sum_{x \in A \cap \Delta_{N}}\left(\xi_{0}^{-l_{x}}\left|\partial A_{x}\right|^{1-1 / \beta}\left|A_{x}\right|^{1 / \beta}\right)^{\alpha(\beta / \alpha)^{\prime}}\right]^{1-\alpha / \beta} } \\
= & {\left[\frac{1}{\left|\Delta_{N}\right|} \sum_{x \in A \cap \Delta_{N}}\left(\xi_{0}^{-l_{x}}\left|\partial A_{x}\right|^{1-1 / \beta}\left|A_{x}\right|^{1 / \beta}\right)^{\alpha \beta /(\beta-\alpha)}\right]^{1-\alpha / \beta} } \\
& \times\left(\sum_{x \in A \cap \Delta_{N}} \frac{\left.\left|\Delta_{N}\right|^{1 / \beta}|| \Phi_{N} 1_{\partial A_{x}} \|_{\Delta_{N}, \beta}\right)^{\beta / \beta}}{\left|A_{N}\right|^{1 / \beta} 1_{\partial A_{x}} \|_{\Delta_{N}, \beta}^{\beta}}\right. \\
\left|A_{x}\right| & { }^{\alpha / \beta}
\end{aligned}
$$

where we used (16) in the first inequality and Hölder's inequality in the second.

Observe that

$$
\sum_{x \in A \cap \Delta_{N}} \frac{\left\|\Phi_{N} 1_{\partial A_{x}}\right\|_{\Delta_{N}, \beta}^{\beta}}{\left|A_{x}\right|} \leq \sum_{i=1}^{n}\left\|\Phi_{N} 1_{\partial A_{i}}\right\|_{\Delta_{N}, \beta}^{\beta} \leq 2 d\left\|\Phi_{N} 1_{\partial A}\right\|_{\Delta_{N}, \beta}^{\beta} \leq C \varepsilon_{0}^{-\beta},
$$


where $A_{1}, \cdots, A_{n}$ are different clusters that intersect with $\Delta_{N}$. On the other hand, the multidimensional ergodic theorem gives

$$
\begin{aligned}
& \lim _{N \rightarrow \infty} \frac{1}{\left|\Delta_{N}\right|} \sum_{x \in A \cap \Delta_{N}}\left(\xi_{0}^{-l_{x}}\left|\partial A_{x}\right|^{1-1 / \beta}\left|A_{x}\right|^{1 / \beta}\right)^{\alpha \beta /(\beta-\alpha)} \\
& =E\left(\xi_{0}^{-l_{o}}\left|\partial A_{o}\right|^{1-1 / \beta}\left|A_{o}\right|^{1 / \beta}\right)^{\alpha \beta /(\beta-\alpha)} \leq C E\left(\xi_{0}^{-l_{o}} l_{o}^{d}\right)^{\alpha \beta /(\beta-\alpha)} \quad \text { P-a.s., }
\end{aligned}
$$

which by (15) is finite when $\varepsilon_{0}$ is small.

\section{Transience in general ergodic environments}

In this section we will prove (ii) of Theorem 1 by an argument similar to [20]. The main differences in our method are that we use a stronger control of the hitting time (Lemma 11), and that we apply a mean value inequality (Theorem 12) instead of the discrete Harnack inequality used in [20].

Lemma 11. Let $\left\{X_{n}\right\}$ be a random walk in a balanced environment $\omega$ such that $\omega(x, o)=0$ for all $x$. For any $r>0$, define $\tau=\tau(r)=\inf \left\{n:\left|X_{n}\right|>\right.$ $r\}$. Then $E_{\omega}^{o} \tau \leq(r+1)^{2}$.

Proof: Observe that $\left\{\left|X_{n}\right|^{2}-n\right\}$ is a (quenched) martingale with respect to $\left\{\mathcal{F}_{n}=\sigma\left(X_{1}, \cdots, X_{n}\right)\right\}$. Thus by optional stopping, $0=E_{\omega}^{o}\left[\left|X_{\tau}\right|^{2}-\tau\right] \leq$ $(r+1)^{2}-E_{\omega}^{o} \tau$.

To prove Theorem 1(ii), we shall make use of the following mean-value inequality, which is a modification of Theorem 3.1 in [11. Let $B_{r}(z)=\{x \in$ $\left.\mathbb{Z}^{d}:|x-z|<r\right\}$. We shall also write $B_{r}(o)$ as $B_{r}$.

Theorem 12. For any function $u$ on $\bar{B}_{R}\left(x_{0}\right)$ such that

$$
L_{\omega} u=0, \quad x \in B_{R}\left(x_{0}\right)
$$

and any $\sigma \in(0,1), 0<p \leq d$, we have

$$
\max _{B_{\sigma R}\left(x_{0}\right)} u \leq C\left\|\frac{u^{+}}{\varepsilon^{d / p}}\right\|_{B_{R}\left(x_{0}\right), p},
$$

where $C$ depends on $\sigma, p$ and $d$.

We postpone the proof of Theorem 12 to the next section, and bring now the

Proof of Theorem 1(ii): As mentioned in Section 1, the transience of the random walk would not change if we considered the walk restricted to its jump times. That is, the transience or recurrence of the random walk in an environment $\omega$ is the same as in an environment $\tilde{\omega}$, where $\tilde{\omega}$ is defined by $\tilde{\omega}(x, e)=\omega(x, e) /(1-\omega(x, o))$. Therefore, in the sequel we assume 
$\omega(x, o)=0$ for all $x$ and almost all $\omega$.

Let $K$ be any constant $\geq 3$. We denote $B_{K^{i}}(x)$ by $B^{i}(x)$ and define $\tau_{i}:=\inf \left\{n:\left|X_{n}\right|>K^{i}\right\}$. Our approach is to bound the (annealed) expected number of visits to the origin by the walk; this requires some a-priori bounds on the moments of $\varepsilon(o)^{-1}$.

For any $z \in \partial B^{i}, y \in B^{i-1}$, noting that $E_{\omega}^{x}$ (\# visits at $y$ before $\left.\tau_{i+2}\right):=$ $v(x)$ satisfies $L_{\omega} v(x)=0$ for $x \in B^{i+2} \backslash\{y\}$, we have that for $p \in(0, d]$,

$$
\begin{aligned}
& E_{\theta^{y} \omega}^{z}\left(\# \text { visits at } o \text { before } \tau_{i+1}\right) \\
& \leq E_{\omega}^{z+y}\left(\# \text { visits at } y \text { before } \tau_{i+2}\right) \\
& \leq \max _{x \in B^{i-1}(z)} E_{\omega}^{x}\left(\# \text { visits at } y \text { before } \tau_{i+2}\right) \\
& \leq C\left\|\frac{E_{\omega}^{x}\left(\# \text { visits at } y \text { before } \tau_{i+2}\right)}{\varepsilon_{\omega}(x)^{d / p}}\right\|_{B_{2 K^{i-1}}(z), p} \\
& \leq C\left\|\frac{E_{\omega}^{x}\left(\# \text { visits at } y \text { before } \tau_{i+2}\right)}{\varepsilon_{\omega}(x)^{d / p}}\right\|_{B^{i+2}, p},
\end{aligned}
$$

where we used Theorem 12 in the third inequality. Take $p=d / q$ (without loss of generality, we always assume that $q<d$ ). Then by (19) and Lemma 11,

$$
\begin{aligned}
& \sum_{y \in B^{i-1}} E_{\theta^{y} \omega}^{o}\left(\# \text { visits at } o \text { in }\left[\tau_{i}, \tau_{i+1}\right)\right) \\
& \leq C \sum_{y \in B^{i-1}}\left[\frac{1}{\left|B^{i+2}\right|} \sum_{x \in B^{i+2}} \frac{E_{\omega}^{x}\left(\# \text { visits at } y \text { before } \tau_{i+2}\right)^{d / q}}{\varepsilon_{\omega}(x)^{d}}\right]^{q / d} \\
& \leq C K^{-i q} \sum_{y \in B^{i-1}} \sum_{x \in B^{i+2}} \frac{E_{\omega}^{x}\left(\# \text { visits at } y \text { before } \tau_{i+2}\right)}{\varepsilon_{\omega}(x)^{q}} \\
& =C K^{-i q} \sum_{x \in B^{i+2}} \frac{E_{\omega}^{x}\left(\# \text { visits at } B^{i-1} \text { before } \tau_{i+2}\right)}{\varepsilon_{\omega}(x)^{q}} \\
& \leq C K^{-i q} \sum_{x \in B^{i+2}} \frac{E_{\omega}^{x} \tau_{i+2}}{\varepsilon_{\omega}(x)^{q}} \\
& \leq C K^{(2-q) i} \sum_{x \in B^{i+2}} \varepsilon_{\omega}(x)^{-q} .
\end{aligned}
$$

Taking expectations and using translation invariance we have

$$
\mathbb{E}^{o}\left(\# \text { visits at } o \text { in }\left[\tau_{i}, \tau_{i+1}\right)\right) \leq C K^{(2-q) i} E \varepsilon^{-q} .
$$

Therefore, if $E \varepsilon^{-q}<\infty$ for some $q>2$, then

$$
\mathbb{E}^{o}(\# \text { visits at } o) \leq C E \varepsilon^{-q} \sum_{i=1}^{\infty} K^{(2-q) i}<\infty .
$$


This proves Theorem 1(ii) for $\{\Omega, P\}$ such that $\omega(x, o)=0$ for all $x$ and almost all $\omega$. As mentioned earlier, the general case follows by replacing $\varepsilon$ with $\varepsilon /(1-\omega(o, o))$.

Remark 13. It is natural to expect that arguments similar to the proof of the invariance principle also work for proving the transience in the i.i.d. case. Namely, one may hope to control $P_{\omega}^{x}\left\{\right.$ visit o in $\left.\left[\tau_{i}, \tau_{i+1}\right)\right\}$ using some mean value inequality (like Theorem 12), and to use percolation arguments to handle "bad sites" where the ellipticity constant $\varepsilon$ is small.

This suggests considering walks that jump from bad sites to good sites. In [12], Kuo and Trudinger proved maximum principle and mean value inequality for balanced operators in general meshes, which may be applied to balanced walks with possibly big jumps. However, their estimates in the presence of a small ellipticity constant are not strong enough. To overcome this issue, we will prove a modified maximum principle that involves only big exit probabilities, and then use it to prove the transience in the i.i.d case with no moment assumptions.

\section{Transience in i.i.d. environment}

In this section we prove a modified maximum principle for balanced environments. We then prove Theorem 2(ii) using the corresponding mean value inequality (Theorem 16) and percolation arguments.

\subsection{Balanced difference operators}

Following [12], we introduce general balanced difference operators. Let $a$ be a nonnegative function on $\mathbb{Z}^{d} \times \mathbb{Z}^{d}$ such that for any $x, a(x, y)>0$ for only finitely many $y$. Define the linear operator $L_{a}$ acting on the set of functions on $\mathbb{Z}^{d}$ by

$$
L_{a} f(x)=\sum_{y} a(x, y)(f(y)-f(x)) .
$$

We say that $L_{a}$ is balanced if

$$
\sum_{y} a(x, y)(y-x)=0 .
$$

Throughout this section we always assume that $L_{a}$ is a balanced probability operator, that is,

$$
\sum_{y} a(x, y)=1
$$

For any finite subset $E \subset \mathbb{Z}^{d}$, define its boundary

$$
E^{b}=E^{b}(a)=\{y \notin E: a(x, y)>0 \text { for some } x \in E\},
$$


and set

$$
\tilde{E}=E \cup E^{b} .
$$

Define the upper contact set of $u$ at $x \in E$ as

$$
I_{u}(x)=I_{u}(x, E, a)=\left\{s \in \mathbb{R}^{d}: u(x)-s \cdot x \geq u(z)-s \cdot z \text { for all } z \in \tilde{E}\right\} .
$$

Set

$$
h_{x}=h_{x}(a)=\max _{y: a(x, y)>0}|x-y| .
$$

The following lemma is useful in the proofs of various mean value inequalities. It is similar to Theorem 2.2 in [12], except that the proof in [12] contains several unclear passages, e.g. in the inequality above (2.23) in [12], and so we provide a complete proof. Throughout, we set $u^{+}=u \vee 0$.

Lemma 14. Fix $R>0$. Let $\eta(x)=\eta_{R}(x):=\left(1-|x|^{2} / R^{2}\right)^{\beta} 1_{|x|<R}$ be a function on $\mathbb{R}^{d}$. For any function $u$ on $B_{R}$ such that $L_{a} u=0$ in $B_{R}$ and any $\beta \geq 2$, we let $v=\eta u^{+}$. Then for any $x \in B_{R}$ with $I_{v}(x)=I_{v}\left(x, B_{R}, a\right) \neq \emptyset$,

$$
L_{a} v(x) \geq-C(\beta) \eta^{1-2 / \beta} R^{-2} h_{x}^{2} u^{+},
$$

where $C(\beta)$ is a constant that depends only on $\beta$.

Proof: We only need to consider the nontrivial case that $v \not \equiv 0$. For $s=s(x) \in I_{v}(x) \neq \emptyset$, recalling the definition of $I_{v}$ one has that

$$
|s| \leq 2 v(x) /(R-|x|) .
$$

Note that $I_{v}(x) \neq \emptyset$ implies $u(x)>0$. If further $R^{2}-|x|^{2} \geq 4 R|x-y|$, computations as in [12, pg. 426] reveal that

$$
\begin{aligned}
2^{-\beta} & \leq \frac{\eta(y)}{\eta(x)} \leq 2^{\beta}, \\
|\eta(x)-\eta(y)| & \leq \beta 2^{\beta} R^{-1} \eta(x)^{1-1 / \beta}|x-y|, \\
|\eta(x)-\eta(y)-\nabla \eta(x)(x-y)| & \leq \beta(\beta-1) 2^{\beta} R^{-2} \eta(x)^{1-2 / \beta}|x-y|^{2}, \\
|s| & \leq 4 \eta^{1-1 / \beta} R^{-1} u,
\end{aligned}
$$

where $\nabla \eta$ is the gradient of $\eta$. Following [12, we set $w(z)=v(z)-s \cdot(z-x)$. By the definition of $s$, we have $w(x) \geq w(z)$ for all $z \in \tilde{E}$ and

$$
\begin{aligned}
& \sum_{y} a(x, y)(v(x)-v(y)) \\
& \stackrel{217}{=} \sum_{y} a(x, y)(w(x)-w(y))
\end{aligned}
$$

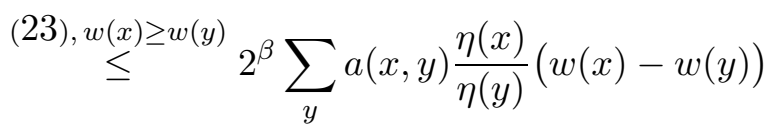

$$
\begin{aligned}
& =2^{\beta} \sum_{y} a(x, y)\left[\frac{\eta(x)}{\eta(y)}(v(x)-v(y))+\frac{\eta(x)}{\eta(y)} s(y-x)\right] .
\end{aligned}
$$


Consider first $x$ such that $R^{2}-|x|^{2} \geq 4 R h_{x}$. Then (recalling that $u(x)>0$ because $\left.I_{v}(x) \neq \emptyset\right)$,

$$
\begin{aligned}
& \sum_{y} a(x, y) \frac{\eta(x)}{\eta(y)}(v(x)-v(y)) \\
& =\sum_{y} a(x, y)\left[\eta(x)\left(u(x)-u^{+}(y)\right)+(\eta(x)-\eta(y)) u(x)+\frac{(\eta(x)-\eta(y))^{2}}{\eta(y)} u(x)\right] \\
& \stackrel{a \geq 0}{\leq} \eta(x) L_{a} u(x)+\sum_{y} a(x, y)\left[(\eta(x)-\eta(y)) u(x)+\frac{(\eta(x)-\eta(y))^{2}}{\eta(y)} u(x)\right] \\
& \stackrel{L_{a} u=0, \text { (21) }}{=} \sum_{y} a(x, y)\left[(\eta(x)-\eta(y)-\nabla \eta(x)(x-y)) u(x)+\frac{(\eta(x)-\eta(y))^{2}}{\eta(y)} u(x)\right] \\
& \leq \beta^{2} 2^{3 \beta+1} \eta^{1-2 / \beta} R^{-2} h_{x}^{2} u,
\end{aligned}
$$

where we used (23), (24), (25) in the last inequality. Moreover, by (21), (23), (24) and (26),

$$
\begin{aligned}
\sum_{y} a(x, y) \frac{\eta(x)}{\eta(y)} s(y-x) & =\sum_{y} a(x, y) \frac{\eta(x)-\eta(y)}{\eta(y)} s \cdot(y-x) \\
& \leq \beta 2^{2 \beta+2} \eta^{1-2 / \beta} R^{-2} h_{x}^{2} u .
\end{aligned}
$$

Hence, combining (27), (28) and (29), we conclude that

$$
-L_{a} v \leq \beta^{2} 2^{4 \beta+2} \eta^{1-2 / \beta} R^{-2} h_{x}^{2} u
$$

holds in $\left\{x: R^{2}-|x|^{2} \geq 4 R h_{x}, I_{v}(x) \neq \emptyset\right\}$.

On the other hand, if $R^{2}-|x|^{2}<4 R h_{x}$, then $\eta^{1 / \beta} \leq 4 h_{x} / R$. Thus by the fact that $v \geq 0$, we have $-L_{a} v \leq 2 v(x) \leq 32 \eta^{1-2 / \beta} R^{-2} h_{x}^{2} u$.

Proof of Theorem 12: Since $L_{\omega}$ is a balanced operator and $h_{x}=1$ in this case, by the above lemma,

$$
L_{\omega} v \geq-C(\beta) \eta^{1-2 / \beta} R^{-2} u
$$

for $x \in B_{R}$ such that $I_{u}(x) \neq \emptyset$. Applying Theorem 3 to $v$ and taking $\beta=2 d / p \geq 2$, we obtain

$$
\begin{aligned}
\max _{B_{R}} v & \leq C\left\|\eta^{1-2 / \beta} \frac{u^{+}}{\varepsilon}\right\|_{B_{R}, d}=C\left\|v^{1-p / d} \frac{\left(u^{+}\right)^{p / d}}{\varepsilon}\right\|_{B_{R}, d} \\
& \leq C\left(\max _{B_{R}} v\right)^{1-p / d}\left\|\frac{u^{+}}{\varepsilon^{d / p}}\right\|_{B_{R}, p}^{p / d} .
\end{aligned}
$$

Hence

$$
\max _{B_{R}} v \leq C\left\|\frac{u^{+}}{\varepsilon^{d / p}}\right\|_{B_{R}, p},
$$


and then

$$
\max _{B_{\sigma R}} u \leq\left(1-\sigma^{2}\right)^{-2 d / p} \max _{B_{\sigma R}} v \leq C(\sigma, p, d)\left\|\frac{u^{+}}{\varepsilon^{d / p}}\right\|_{B_{R}, p} .
$$

\subsection{A new maximum principle and proof of Theorem 2 (ii)}

For any fixed environment $\omega \in \Omega$, let $\varepsilon_{0}>0$ be a constant to be determined, and define site percolation as in Section 4. Recall that for $x \in \mathbb{Z}^{d}, A_{x}$ is the percolation cluster that contains $x$ and $l_{x}$ is its $l^{1}$-diameter. As mentioned in the introduction, the transience would not change if we considered the walk restricted to its jump times. Without loss of generality, we assume that $\omega(x, o)=0$ for all $x, P$-almost surely.

Recall the definition of $\square$ and $\kappa$-path for $\kappa \in \square$ in Section 4. Note that under our assumption, $\max _{i} \omega\left(x, e_{i}\right) \geq 1 / 2 d$, so we take $\xi_{0}=1 / 2 d$ in the definition of $\kappa$-paths.

For each $\kappa \in \square$, we pick a site $y_{\kappa}=y(x, \kappa) \in \partial A_{x}$ such that

$$
d\left(x, y_{\kappa}\right)=\max _{y: \exists \text { m-path in } \bar{A}_{x}} d(x, y)
$$

and let $\Lambda_{x} \subset \bar{A}_{x}$ be the union of (the points of the) $\kappa$-paths from $x$ to $y_{\kappa}$ over all $\kappa \in \square$. From the definition of $y_{\kappa}$ one can conclude that

- For any $q \in \mathbb{R}^{d}$, we pick a $\kappa=\kappa_{q} \in \square$ such that

$$
q_{j} \kappa_{j} \leq 0 \text { for all } j=1, \cdots, d .
$$

Then $\left(y_{\kappa}-x\right)_{j} q_{j} \leq 0$ for all $j=1, \cdots, d$. Moreover, for $i \in\{1, \cdots, d\}$, $q_{i}>0$ implies $y_{\kappa}-e_{i} \notin \Lambda_{x}$, and $q_{i}<0$ implies $y_{\kappa}+e_{i} \notin \Lambda_{x}$.

In the sequel we let $\tau_{\Lambda_{x}}=\inf \left\{n>0: X_{n} \notin \Lambda_{x}\right\}$ and

$$
a(x, y)=P_{\omega}^{x}\left\{X_{\tau_{\Lambda_{x}}}=y\right\} .
$$

By the fact that $X_{n}$ is a (quenched) martingale, it follows that $L_{a}$ is a balanced operator.

For the statement of the next theorem, recall the definition of $\tilde{E}$, see (22).

Theorem 15. Let $E \subset \mathbb{Z}^{d}$ be bounded. Let $u$ be a function on $\tilde{E}$. If $L_{a} u(x) \geq-g(x)$ for all $x \in E$ such that $I_{u}(x)=I_{u}(x, E, a) \neq \emptyset$, then

$$
\max _{E} u \leq \frac{d \operatorname{diam} \tilde{E}}{\varepsilon_{0}}\left(\sum_{\substack{x \in E \\ I_{u}(x) \neq \emptyset}}\left|g(x)(2 d)^{l_{x}}\right|^{d}\right)^{\frac{1}{d}}+\max _{E^{b}} u .
$$


Proof: Without loss of generality, assume $g \geq 0$ and

$$
\max _{E} u=u\left(x_{0}\right)>\max _{E^{b}} u
$$

for some $x_{0} \in E$. Otherwise, there is nothing to prove.

For $s \in \mathbb{R}^{d}$ such that $|s|_{\infty} \leq\left[u\left(x_{0}\right)-\max _{E^{b}} u\right] /(d \operatorname{diam} \tilde{E})$, we have

$$
u\left(x_{0}\right)-u(x) \geq s \cdot\left(x_{0}-x\right)
$$

for all $x \in E^{b}$, which implies that $\max _{z \in \tilde{E}} u(z)-s \cdot z$ is achieved in $E$. Hence $s \in \bigcup_{x \in E} I_{u}(x)$ and

$$
\left[-\frac{u\left(x_{0}\right)-\max _{E^{b}} u}{d \operatorname{diam} \tilde{E}}, \frac{u\left(x_{0}\right)-\max _{E^{b}} u}{d \operatorname{diam} \tilde{E}}\right]^{d} \subset \bigcup_{x \in E} I_{u}(x) .
$$

Further, if $s \in I_{u}(x)$, we set

$$
w(z)=u(z)-s(z-x) .
$$

Then $w(z) \leq w(x)$ for all $z \in \tilde{E}$ and

$$
I_{u}(x)=I_{w}(x)+s .
$$

Since for any $q \in I_{w}(x)$, there is $\kappa=\kappa_{q} \in \square$ such that

$$
q_{j}\left(x-y_{\kappa}\right)_{j} \geq 0 \text { for } j=1, \cdots, d,
$$

we have

$$
w(x)-w\left(y_{\kappa} \pm e_{i}\right) \geq q\left(x-y_{\kappa} \mp e_{i}\right) \geq \mp q_{i} .
$$

Moreover, for any $i \in\{1, \cdots, d\}$, if $q_{i}>0$, then $y_{\kappa}-e_{i} \notin \Lambda_{x}$ and we have $w(x)-w\left(y_{\kappa}-e_{i}\right) \geq\left|q_{i}\right|$. Similarly, if $q_{i}<0$, then $y_{\kappa}+e_{i} \notin \Lambda_{x}$ and $w(x)-w\left(y_{\kappa}+e_{i}\right) \geq\left|q_{i}\right|$. We conclude that

$$
\left|q_{i}\right| \leq \frac{\sum_{y} a(x, y)(w(x)-w(y))}{\min _{ \pm}\left\{a\left(x, y_{\kappa} \pm e_{i}\right)\right\}}
$$

On the other hand, from the construction of $\Lambda_{x}$ we obtain (note that $y_{\kappa} \in$ $\left.\partial A_{x}\right)$

$$
a\left(x, y_{\kappa} \pm e_{i}\right) \geq\left(\frac{1}{2 d}\right)^{l_{x}} \varepsilon_{0} .
$$

Hence, since $L_{a}$ is balanced,

$$
\left|q_{i}\right| \leq \frac{(2 d)^{l_{x}}}{\varepsilon_{0}} \sum_{y} a(x, y)(w(x)-w(y))=\frac{(2 d)^{l_{x}}}{\varepsilon_{0}}\left(-L_{a} u\right) \leq \frac{(2 d)^{l_{x}}}{\varepsilon_{0}} g
$$

for all $i$. Therefore

$$
I_{w}(x) \subset\left[-(2 d)^{l_{x}} \varepsilon_{0}^{-1} g,(2 d)^{l_{x}} \varepsilon_{0}^{-1} g\right]^{d} .
$$


Combining (30), (31) and (32) we conclude that

$$
\left(\frac{u\left(x_{0}\right)-\max _{E^{b}} u}{d \operatorname{diam} \tilde{E}}\right)^{d} \leq \sum_{\substack{x \in E \\ I_{u}(x) \neq \emptyset}}\left|g(x)(2 d)^{l_{x}} \varepsilon_{0}^{-1}\right|^{d} .
$$

As with Theorem [12, we have a corresponding mean value inequality.

Theorem 16. For any function $u$ on $B_{R}$ such that

$$
L_{a} u=0, \quad x \in B_{R}
$$

and any $\sigma \in(0,1), 0<p \leq d$, we have

$$
\max _{B_{\sigma R}} u \leq C\left(\frac{\operatorname{diam} \tilde{B}_{R}}{\varepsilon_{0} R}\right)^{d / p}\left\|\left[l_{x}^{2}(2 d)^{l_{x}}\right]^{d / p} u^{+}\right\|_{B_{R}, p},
$$

where $C$ depends on $\sigma, p$ and $d$.

Proof: By the same argument as in the proof of Theorem 12, Lemma 14 and Theorem 15 implies Theorem 16.

Having established Theorem 16, we can now prove the transience of the random walks in balanced i.i.d. environment with $d \geq 3$.

Proof of Theorem Q(ii): Let $K$ be any constant $\geq 4$ and define $B^{i}, \tau_{i}$ as in Section 5. Let $\Omega_{i}=\left\{\omega \in \Omega: l_{x} \leq K^{i-1}\right.$ for all $\left.x \in B^{i+2}\right\}$. For any $\omega \in \Omega_{i}$, $z \in \partial B^{i}, y \in B^{i-1}$, noting that $P_{\omega}^{x}\left\{\right.$ visit $y$ before $\left.\tau_{i+2}\right\}:=u(x)$ satisfies

$$
L_{a} u(x)=0
$$

for $x \in B_{2 K^{i-1}}(z)$, by similar argument as in (19) we have

$$
\begin{aligned}
& P_{\theta^{y} \omega}^{z}\left\{\text { visit } o \text { before } \tau_{i+1}\right\} 1_{\omega \in \Omega_{i}} \\
& \leq \max _{x \in B^{i-1}(z)} P_{\omega}^{x}\left\{\text { visit } y \text { before } \tau_{i+2}\right\} 1_{\omega \in \Omega_{i}} \\
& \leq C \varepsilon_{0}^{-d} \|\left[l_{x}^{2}(2 d)^{l_{x}}\right]^{d} P_{\omega}^{x}\left\{\text { visit } y \text { before } \tau_{i+2}\right\} \|_{B_{2 K^{i-1}}(z), 1} \\
& \leq C \varepsilon_{0}^{-d}\left|B^{i+2}\right|^{-1} \sum_{x \in B^{i+2}} l_{x}^{2 d}(2 d)^{d l_{x}} P_{\omega}^{x}\left\{\text { visit } y \text { before } \tau_{i+2}\right\},
\end{aligned}
$$

where in the second inequality, we applied Theorem 16 with $p=1$ and used the fact that $\operatorname{diam} \tilde{B}_{2 K^{i-1}} \leq 3 K^{i-1}$ when $\omega \in \Omega_{i}$. Hence

$$
\begin{aligned}
& \sum_{y \in B^{i-1}} P_{\theta^{y}}^{o}\left\{\text { visit } o \text { in }\left[\tau_{i}, \tau_{i+1}\right)\right\} 1_{\omega \in \Omega_{i}} \\
& \leq C \varepsilon_{0}^{-d}\left|B^{i+2}\right|^{-1} \sum_{x \in B^{i+2}} l_{x}^{2 d}(2 d)^{d l_{x}} E_{\omega}^{x}\left(\# \text { visits at } B^{i-1} \text { before } \tau_{i+2}\right) \\
& \underset{\leq}{\text { Lemma } 11} C \varepsilon_{0}^{-d} K^{(2-d) i} \sum_{x \in B^{i+2}} l_{x}^{2 d}(2 d)^{d l_{x}} .
\end{aligned}
$$


Since

$$
\begin{aligned}
& \sum_{y \in B^{i-1}} P_{\theta^{y} \omega}^{o}\left\{\text { visit } o \text { in }\left[\tau_{i}, \tau_{i+1}\right)\right\} \\
& \leq \sum_{y \in B^{i-1}} P_{\theta^{y} \omega}^{o}\left\{\text { visit } o \text { in }\left[\tau_{i}, \tau_{i+1}\right)\right\} 1_{\omega \in \Omega_{i}}+\left|B^{i-1}\right| 1_{\omega \notin \Omega_{i}},
\end{aligned}
$$

taking $P$-expectations on both sides of (34) and using (33) we get

$$
\mathbb{P}^{o}\left\{\text { visit } o \text { in }\left[\tau_{i}, \tau_{i+1}\right)\right\} \leq C \varepsilon_{0}^{-d} K^{(2-d) i} E l_{o}^{2 d}(2 d)^{d l_{o}}+P\left\{\omega \notin \Omega_{i}\right\} .
$$

By (15), we can take $\varepsilon_{0}$ to be small enough such that $E l_{o}^{2 d}(2 d)^{d l_{o}}<\infty$ and $\sum_{i=1}^{\infty} P\left\{\omega \notin \Omega_{i}\right\}<\infty$. Therefore when $d \geq 3$,

$$
\sum_{i=1}^{\infty} \mathbb{P}^{o}\left\{\text { visit } o \text { in }\left[\tau_{i}, \tau_{i+1}\right)\right\}<\infty
$$

\section{Concluding remark}

While Bouchaud's trap model (see [5, 3]) provides an example of an (i.i.d.) environment where local traps can destroy the invariance principle, it is interesting to note that a counter-example to Theorem 2 in the ergodic setup also can be written. Namely, let $d \geq 2$, write for $x \in \mathbb{Z}^{d}, z(x)=$ $\left(x_{2}, \cdots, x_{d}\right) \in \mathbb{Z}^{d-1}$. Let $\left\{\varepsilon_{z}\right\}_{z \in \mathbb{Z}^{d-1}}$ be i.i.d random variables with support in $(0,1 / 2)$ and set

$$
\omega(x, e)=\left\{\begin{aligned}
\varepsilon_{z(x)}, & \text { if } e= \pm e_{1} \\
\left(1-2 \varepsilon_{z(x)}\right) / 2(d-1), & \text { else }
\end{aligned}\right.
$$

It is easy to verify that $\left\{X_{t}^{n}\right\}_{t \geq 0}$ satisfies the quenched invariance principle, but that the limiting covariance may degenerate if the tail of $\varepsilon_{z}$ is heavy.

\section{References}

[1] M. Barlow, Random walks on supercritical percolation clusters, Annals Probab. 32 (2004), 3024-3084.

[2] M. Barlow, J.-D. Deuschel, Invariance principle for the random conductance model with unbounded conductances, Annals Probab. 38 (2010), 234-276.

[3] G. Ben Arous, J. Cerny, Scaling limits for trap models on $\mathbb{Z}^{D}$, Annals Probab. 35 (2007), 2356-2384.

[4] N. Berger, M. Biskup, Quenched invariance principle for simple random walks on percolation clusters, Probab. Theory Related Fields 137 (2007), 83-120. 
[5] J.P.Bouchaud, Weak ergodicity breaking and aging in disordered systems, J. Phys. I (France) 2, 1705 (1992).

[6] A. De Masi, P. A. Ferrari, S. Goldstein, W. D. Wick, An invariance principle for reversible Markov processes. Applications to random motions in random environments, J. Statist. Phys. 55 (1989), 787-855.

[7] M. Fekete, Über die Verteilung der Wurzeln bei gewissen algebraischen Gleichungen mit ganzzahligen Koeffizienten, Mathematische Zeitschrift 17 (1923), 228-249.

[8] H. O. Georgii, Gibbs Measures and Phase Transitions, Walter de Gruyter, Berlin, 1988.

[9] G. Grimmett, Percolation (Second edition), Springer, 1999.

[10] U. Krengel, Ergodic theorems, Walter de Gruyter, Berlin, 1985.

[11] H. J. Kuo, N. S. Trudinger, Linear elliptic difference inequalities with random coefficients, Math. Comp. 55 (1990), 37-53.

[12] H. J. Kuo, N. S. Trudinger, Positive difference operators on general meshes, Duke Math. J. 83 (1996), 415-433.

[13] G. F. Lawler, Weak convergence of a random walk in a random environment, Comm. Math. Phys. 87 (1982), 81-87.

[14] P. Mathieu, Quenched invariance principles for random walks with random conductances, J. Stat. Phys. 130 (2008), 1025-1046.

[15] P. Mathieu, E. Remy, Isoperimetry and heat kernel decay on percolation clusters, Annals Probab. 32 (2004), 100-128.

[16] P. Mathieu, A. Pianitski, Quenched invariance principles for random walks on percolation clusters, Proc. R. Soc. Lond. Ser. A Math. Phys. Eng. Sci. 463 (2007), 2287-2307.

[17] G. Papanicolaou, S.R.S.Varadhan, Diffusions with random coefficients, Statistics and probability: essays in honor of C. R. Rao, North-Holland, Amsterdam, 1982, pp. 547-552

[18] A. S. Sznitman, Lectures on random motions in random media, In DMV seminar 32, Birkhauser, Basel, 2002.

[19] V. Sidoravicius, A. S. Sznitman, Quenched invariance principles for walks on clusters of percolation or among random conductances, Probab. Theory Related Fields 129 (2004), 219-244. 
[20] O. Zeitouni, Random walks in random environment, XXXI Summer school in Probability, St. Flour (2001). Lecture notes in Math. 1837, Berlin:Springer, 2004, 193-312

[21] O. Zeitouni, Random walks in random environments, J. Phys. A 39 (2006), R433-R464. 